\title{
Sialyl Lewis X Expression and Lymphatic Microvessel Density in Primary Tumors of Node-negative Colorectal Cancer Patients Predict Disease Recurrence
}

\author{
Fania S. Doekhie • Hans Morreau • \\ Geertruida H. de Bock • Frank M. Speetjens • \\ N. Geeske Dekker-Ensink • Hein Putter • \\ Cornelis J. H. van de Velde • Rob A. E. M. Tollenaar • \\ Peter J. K. Kuppen
}

Received: 28 May 2008 / Accepted: 17 July 2008 /Published online: 6 August 2008

(C) Springer Science + Business Media B.V. 2008

\begin{abstract}
Up to $30 \%$ of curatively resected colorectal cancer patients with tumor-negative lymph nodes, show disease recurrence. We assessed whether these high-risk patients can be identified by examining primary tumors for the following blood and lymphatic vasculature markers: A)
\end{abstract}

Fania S. Doekhie was Supported by the Dutch Cancer Society (project number 2000-2211)

F. S. Doekhie $\cdot$ F. M. Speetjens $\cdot$ N. G. Dekker-Ensink $\cdot$

C. J. H. van de Velde • R. A. E. M. Tollenaar •

P. J. K. Kuppen $(\square)$

Department of Surgery, K6-R, Leiden University Medical Center, P.O. Box 9600, 2300 RC Leiden, The Netherlands

e-mail: p.j.k.kuppen@lumc.nl

\section{F. S. Doekhie}

Department of Surgery, Rijnstate Hospital,

Arnhem, The Netherlands

H. Morreau

Department of Pathology,

Leiden University Medical Center,

Leiden, The Netherlands

G. H. de Bock

Department of Medical Decision Making,

Leiden University Medical Center,

Leiden, The Netherlands

G. H. de Bock

Department of Epidemiology,

Groningen University Medical Center, University of Groningen,

Groningen, The Netherlands

H. Putter

Department of Medical Statistics and Bioinformatics,

Leiden University Medical Center,

Leiden, The Netherlands sialyl Lewis X (sLeX), vascular endothelial growth factor (VEGF)-C and VEGF-D expression; B) blood and lymphatic microvessel density (BMVD/LMVD); and C) the presence of blood and lymphatic vessel invasion. Thirty-six cases (disease recurrence within 5 years) and 72 controls (no disease recurrence for at least 5 years) were selected in a case-control design. Tumor sections were stained by antibodies CSLEX1 (sLeX), anti-VEGF-C, anti-VEGF-D, anti-CD31 (BMVD) or D2-40 (LMVD) to determine the parameters as mentioned above. A multivariate analysis showed sLeX expression and high LMVD (odds ratio 5.1, 95\% confidence interval $1.3-20.0$ and odds ratio $3.1,95 \%$ confidence interval 1.0-10.0, respectively) to be independent factors predicting disease recurrence. Expression of sLeX correlated with liver metastases $(P=0.015)$. A high LMVD was related to regional intra-abdominal or intrapelvic metastases in lymph nodes and distant metastases other than in the liver and lungs such as peritoneum, bones, brain and adrenal glands $(P=0.004)$. A high BMVD in the invasive front correlated with lung metastases $(P=0.018)$. We show that high-risk node-negative colorectal cancer patients can be identified by primary tumor assessment for sLeX expression and LMVD. Our results are consistent with the notion that both lymphatic and hematogenous metastasis play a role in colorectal cancer.

Keywords Angiogenesis - Colorectal cancer -

Disease recurrence $\cdot$ Lymphangiogenesis .

Microvessel density · Sialyl Lewis X ·

Vascular endothelial growth factor - Vessel invasion .

CD31 - D2-40 


$\begin{array}{ll}\text { Abbreviations } \\ \text { BMVD } & \text { blood microvessel density } \\ \text { CRC } & \text { colorectal cancer } \\ \text { LMVD } & \text { lymphatic microvessel density } \\ \text { SLeX } & \text { sialyl Lewis X } \\ \text { VEGF } & \text { vascular endothelial growth factor }\end{array}$

\section{Introduction}

In most countries, patients with curatively resected colorectal cancer (CRC) without nodal tumor involvement do not receive adjuvant systemic therapy since meaningful survival benefits have not been proven yet $[1,2]$. However, 10 to 30 percent of these patients show disease recurrence [3, 4]. Although, a small survival benefit has been reported in stage II CRC patients who received 5-fluorouracil [5], adjuvant systemic treatment of all node-negative CRC patients will lead to overtreatment and unnecessary complications due to adverse effects of the treatment. Providing only high-risk patients with adjuvant systemic therapy prevents unnecessary treatment and leads to an optimized cure rate. Lymph node-negative CRC patients at risk for disease recurrence may be identified by tumor features known to determine the process of invasion and metastasis which was the focus of this study.

Invasion and metastasis can be facilitated by proteins which stimulate tumor cell attachment to host cellular or extracellular matrix determinants, proteolysis of extracellular matrix host barriers such as the basement membrane, tumor cell migration and formation of distant metastases [6]. Tumor cells are expected to express similar molecules as leucocytes to interact with blood and lymphatic microvessel endothelium. Normally, the blood group-related antigen sialyl Lewis $\mathrm{X}(\mathrm{sLeX})$ is located on the membrane of neutrophils and binds to the endothelial leukocyte adhesion molecule-1 also known as E-selectin which leads to neutrophil extravasation and migration into tissue [7]. In cancer, the interaction of sLeX on tumor cells and Eselectin was shown to mediate adhesion of tumor cells to endothelial cells [8]. Previous publications have reported high sLeX expression on tumor cells to be a prognostic factor in colorectal cancer $[9,10]$.

The vascular endothelial growth factor (VEGF) family has been reported to be involved in the formation of blood and lymphatic microvessels [11] through which tumor cells disseminate. The VEGF family includes five ligands: VEGF-A, VEGF-B, VEGF-C, VEGF-D, and placenta growth factor and three receptors: VEGFR-1, VEGFR-2, and VEGFR-3 [12]. Studies have shown that VEGF-C and VEGF-D, which are ligands for VEGFR-3, can induce tumor lymphangiogenesis and lymphatic metastasis $[13,14]$.

Also, the number and tumor invasion of blood and lymphatic microvessels have been shown to play a role in the process of metastasis [15-17].

In this study, we examined whether disease recurrence in lymph node-negative CRC patients could be predicted by assessing their primary tumors for: A) the expression of angiogenic and lymphangiogenic factors, sLeX, VEGF$\mathrm{C}$, and VEGF-D; B) blood and lymphatic microvessel density; and C) the presence of blood and lymphatic vessel invasion.

\section{Materials and Methods}

\section{Patients}

Between January 1981 and December 2001, 1044 patients underwent surgery for a primary CRC at the LUMC. For the present study, a selection was made from the total group of patients with tumor-negative lymph nodes (N0) and without metastases (M0) at the time of surgery $(n=506)$. Patients who were operated on their first CRC in another hospital, or who were diagnosed with another invasive malignancy before or within 5 years after the date of diagnosis of the primary colorectal carcinoma, and patients who developed a local recurrence were excluded for the present study. Cases $(n=36)$ were defined as patients who suffered from regional or distant recurrent disease at least 3 months after but within 5 years after the date of diagnosis of primary CRC. Cases developed liver metastases $(n=23)$, and/or lung metastases $(n=8)$, and/or regional metastases which were considered intra-abdominal or intrapelvic metastases in lymph nodes or in connective tissue $(n=7)$, and/or peritoneal metastases $(n=5)$, and/or other distant metastases $(n=8)$. The latter consists of brain, bone or adrenal metastases. Controls were patients who did not develop locoregional or distant disease within 5 years after diagnosis of primary CRC. For each case two controls were matched for TNM stage, date of incidence and date of birth, leading to a total number of 72 controls. Of patients with rectal carcinoma, two had received preoperative and three had received postoperative radiotherapy. None of the patients had received adjuvant chemotherapy.

A tumor was considered mucinous when more than $50 \%$ of its volume consisted of mucinous component. Tumor growth pattern at the invasive margin was classified as either expanding also known as pushing or infiltrative based on the predominant morphology as defined by Jass et al. [18] Clinicopathologic characteristics of patients are shown in Table 1. 
Table 1 Patient and primary tumor characteristics

\begin{tabular}{|c|c|c|c|c|c|c|c|}
\hline \multirow[t]{2}{*}{ Characteristics } & \multicolumn{2}{|c|}{ All patients $(n=108)$} & \multicolumn{2}{|c|}{ Cases $(n=36)$} & \multicolumn{2}{|c|}{ Controls $(n=72)$} & \multirow[t]{2}{*}{$\mathrm{P}^{\mathrm{h}}$} \\
\hline & Number & Percent & Number & Percent & Number & Percent & \\
\hline \multicolumn{8}{|l|}{ Sex } \\
\hline Female & 53 & 49 & 16 & 44 & 37 & 51 & \multirow[t]{2}{*}{0.496} \\
\hline Male & 55 & 51 & 20 & 56 & 35 & 49 & \\
\hline Age (years) ${ }^{\mathrm{a}}$ & \multicolumn{2}{|l|}{$67 \pm 12$} & \multicolumn{2}{|l|}{$67 \pm 12$} & \multicolumn{2}{|l|}{$67 \pm 12$} & $0.911^{\mathrm{i}}$ \\
\hline \multicolumn{8}{|l|}{ TNM stage $^{\mathrm{b}}$} \\
\hline I & 6 & 6 & 2 & 6 & 4 & 6 & \multirow[t]{2}{*}{$1.000^{\mathrm{j}}$} \\
\hline II & 102 & 94 & 34 & 94 & 68 & 94 & \\
\hline \multicolumn{8}{|l|}{ T stage ${ }^{c}$} \\
\hline $\mathrm{T} 2$ & 6 & 6 & 2 & 6 & 4 & 6 & \multirow[t]{3}{*}{0.126} \\
\hline $\mathrm{T} 3$ & 88 & 82 & 26 & 72 & 62 & 86 & \\
\hline $\mathrm{T} 4$ & 14 & 13 & 8 & 22 & 6 & 8 & \\
\hline Tumor size $(\mathrm{cm})(n=104)^{\mathrm{a}, \mathrm{d}}$ & \multicolumn{2}{|l|}{$5.1 \pm 2.0$} & \multicolumn{2}{|c|}{$4.9 \pm 1.9(n=34)$} & \multicolumn{2}{|c|}{$5.2 \pm 2.1(\mathrm{n}=70)$} & $0.539^{\mathrm{i}}$ \\
\hline \multicolumn{8}{|l|}{ Tumor location } \\
\hline Colon (coecum-sigmoid) & 89 & 82 & 30 & 83 & 59 & 82 & \multirow[t]{2}{*}{0.858} \\
\hline Rectum (rectosigmoid-rectum) & 19 & 18 & 6 & 17 & 13 & 18 & \\
\hline \multicolumn{8}{|l|}{ Differentiation } \\
\hline Good & 27 & 25 & 9 & 25 & 18 & 25 & \multirow[t]{3}{*}{0.912} \\
\hline Moderate & 68 & 63 & 22 & 61 & 46 & 64 & \\
\hline Poor & 13 & 12 & 5 & 14 & 8 & 11 & \\
\hline \multicolumn{8}{|l|}{ Mucinous } \\
\hline No & 98 & 91 & 32 & 89 & 66 & 92 & \multirow[t]{2}{*}{$0.728^{\mathrm{j}}$} \\
\hline Yes & 10 & 9 & 4 & 11 & 6 & 8 & \\
\hline \multicolumn{8}{|l|}{ Growth pattern $(n=97)^{\mathrm{e}}$} \\
\hline Expanding or pushing & 70 & 72 & 20 & 60 & 50 & 79 & \multirow[t]{2}{*}{0.031} \\
\hline Infiltrating & 27 & 28 & 14 & 41 & 13 & 21 & \\
\hline Number of lymph nodes ${ }^{\mathrm{f}}$ & $6(1-26)$ & & $6(1-18)$ & & $6(1-26)$ & & $0.096^{\mathrm{k}}$ \\
\hline \multicolumn{8}{|c|}{ Preoperative serum CEA level $(n=43)^{\mathrm{g}}$} \\
\hline$<6 \mathrm{ug} / 1$ & 30 & 70 & 5 & 56 & 25 & 74 & \multirow[t]{2}{*}{$0.417^{\mathrm{j}}$} \\
\hline$\geq 6 \mathrm{ug} / 1$ & 13 & 30 & 4 & 44 & 9 & 26 & \\
\hline
\end{tabular}

CEA Carcinoembryonic antigen

${ }^{\text {a }}$ Presented as mean \pm standard deviation

${ }^{\mathrm{b}}$ According to the 6th edition of the TNM classification [39]

${ }^{\mathrm{c}}$ If T2 and T3 stage were combined, the $P$-value was 0.043 ; this comparison was therefore used in the logistic regression

${ }^{\mathrm{d}}$ Tumor size could not be found in pathology reports from four patients

${ }^{\mathrm{e}}$ Patients were excluded from analysis if no invasive front was found in primary tumor sections

${ }^{\mathrm{f}}$ Presented as median and range between brackets

${ }^{g}$ Serum CEA had been determined in only 43 of 108 patients as it was not a standard procedure

${ }^{\mathrm{h}}$ Chi-square test of cases versus controls, unless mentioned otherwise

${ }^{\mathrm{i}}$ Student T-test

${ }^{\mathrm{j}}$ Fisher's exact test

${ }^{\mathrm{k}}$ Mann-Whitney test

\section{Immunohistochemistry}

Four $\mu \mathrm{m}$ sections were cut from formalin-fixed paraffinembedded tumors, transferred to aminopropylethoxysilane(APES) coated slides, and dried overnight at $37^{\circ} \mathrm{C}$. One section of each tumor was stained by hematoxylin and eosin (HE). Five sections of each tumor were respectively stained with CSLEX1 also known as CD15s (Becton Dickinson Pharmingen, San Jose, California, 1:150), a monoclonal antibody that binds to sLeX, polyclonal anti-
VEGF-C (Zymed, San Francisco, California, 1:100), monoclonal anti-VEGF-D (Research and Development Systems, Minneapolis, USA, 1:400), monoclonal antibody anti-CD31 (Dako, Glostrup, Denmark, 1:300), or monoclonal antibody D2-40 (Signet Laboratories, Dedham, MA, USA, 1:100). All primary antibodies were diluted in phosphatebuffered saline (PBS) with $1 \%$ bovine serum albumine (BSA). As negative control the sections were incubated with only $\mathrm{PBS} / 1 \%$ BSA. For the immunohistochemical staining procedure, the sections were deparaffinized in xylene. Endogenous 
peroxidase was blocked in methanol containing $0.3 \%$ hydrogen peroxide at room temperature for $20 \mathrm{~min}$. Subsequently the sections were rehydrated. After washing in PBS, antigen retrieval treatment for the antibodies CSLEX1, anti-VEGF-C and anti-VEGF-D was done by boiling the sections in $1 \mathrm{mM}$ EDTA (ethylene diamine tetra acetic acid, $\mathrm{pH}$ 8.0) solution during $10 \mathrm{~min}$. Antigen retrieval for anti-CD31 and D2-40 was done by boiling the sections in a $0.01 \mathrm{M}$ sodium citrate solution (pH 6.0) during $10 \mathrm{~min}$. After applying the primary antibodies, the sections were incubated overnight at room temperature. Detection of the antigen was achieved by incubating the sections for $30 \mathrm{~min}$ with the biotinylated rabbit-anti-mouse conjugate (Dako, Glostrup, Denmark, 1:200) or with biotinylated swine-anti-rabbit conjugate (Dako, Glostrup, Denmark, 1:400) for VEGF-C and incubation for 30 min with a streptavidin-biotin-peroxidase conjugate (DAKO, Glostrup, Denmark, 1:100). For the staining with anti-CD31 and D2-40, detection of the antigen was achieved with Envision (Dako, Glostrup, Denmark). The sections were developed in 3,3-di-amino-benzidine tetrahydrochloride (DAB) substrate in a buffered $0.05 \mathrm{M}$ Tris/ $\mathrm{HCl}(\mathrm{pH}$ 7.6) solution containing $0.002 \%$ hydrogen peroxide leading to brown-staining of the antigen. Counterstaining was done with Mayer's Haematoxylin. For microscopic analysis the sections were dehydrated and mounted in pertex. Tumor cells positive for sLeX, VEGF-C or VEGF-D and vessels positive for CD31 or D2-40 stained brown.

\section{Quantification}

Slides were evaluated by using a standard light microscope. At least two investigators (Doekhie, Morreau, Speetjens or Dekker) independently examined each staining and discordant cases were re-evaluated to reach consensus. Tissue sections which were too much damaged by the staining procedure were excluded from further analysis, leading to a variable number of examined slides (Tables 2 and 3).

Sialyl LeX staining with CSLEX1 showed immunoreactivity of apical cell membranes, cytoplasm of tumor cells and secretory material in luminal spaces as reported previously [9]. For the sLeX expression the percentage of cytoplasmic stained tumor cells and apical membrane stained tumor cells were separately estimated at $100 \mathrm{x}$ magnification. VEGF-C and VEGF-D expression showed cytoplasmic localization. In these stainings, the percentage of cytoplasmic stained tumor cells was estimated, also at 100x magnification.

Microvessel density was assessed in sections stained by anti-CD31 and D2-40 antibodies, as described previously [15]. Three fields with the highest vascular density (hot spots) were identified both within intratumoral stroma and the invasive front if present, using 50x and 100x magnification. Subsequently, the number of vessels was counted using 200x magnification after applying a $10 \times 10$ grid on the hot spots corresponding to an area of $0.25 \mathrm{~mm}^{2}$. All vessels hitting two of the sides of the grid were included in the count, whereas vessels hitting the other two sides of the grid were excluded from the count, using the principles of Gundersen et al. [19]. Microvessels in the submucosa served as internal controls in assessing the quality of staining for CD31 and D2-40. Any immunoreactive endothelial cell or endothelial cell cluster that was clearly separated from the adjacent microvessels was considered as a single countable microvessel. The occasionally found immunoreactive lymphocytes, macrophages, and plasma cells were excluded on the basis of the staining pattern and

Table 2 Sialyl Lewis X and vascular endothelial growth factor C and D cytoplasmic staining in primary colorectal tumors $(n=99)^{\mathrm{a}}$

\begin{tabular}{|c|c|c|c|c|c|c|c|c|c|c|}
\hline & \multicolumn{2}{|c|}{ All patients } & \multicolumn{2}{|l|}{ Cases } & \multicolumn{2}{|l|}{ Controls } & \multirow[t]{2}{*}{$\mathrm{P}^{\mathrm{b}}$} & \multicolumn{3}{|c|}{ Univariate analysis } \\
\hline & Number & Percent & Number & Percent & Number & Percent & & OR & $95 \% \mathrm{CI}$ & $P$ value \\
\hline \multicolumn{11}{|l|}{ CSLEX1 } \\
\hline Negative & 25 & 25 & 3 & 9 & 22 & 33 & 0.012 & 1 & & \\
\hline Positive & 74 & 75 & 29 & 91 & 45 & 67 & & 4.7 & $(1.3-17.2)$ & 0.019 \\
\hline \multicolumn{11}{|l|}{ VEGF-C } \\
\hline Negative & 67 & 68 & 18 & 56 & 49 & 73 & 0.093 & 1 & & \\
\hline Positive & 32 & 32 & 14 & 44 & 18 & 27 & & 2.1 & $(0.9-5.1)$ & 0.096 \\
\hline \multicolumn{11}{|l|}{ VEGF-D } \\
\hline Negative & 88 & 89 & 26 & 79 & 62 & 94 & $0.039^{\mathrm{c}}$ & 1 & & \\
\hline Positive & 11 & 11 & 7 & 21 & 4 & 6 & & 4.2 & $(1.1-15.5)$ & 0.033 \\
\hline
\end{tabular}

$V E G F$ Vascular endothelial growth factor, $O R$ odds ratio, $C I$ confidence interval

${ }^{a}$ Primary tumors from three controls could not be retrieved from the archive and tissue sections which were destroyed after staining were excluded from analysis leading to a lower number of examined slides

${ }^{\mathrm{b}}$ Chi-square test of cases versus controls, unless mentioned otherwise

${ }^{\mathrm{c}}$ Fisher's exact test 
Table 3 Microvessel density and microvessel tumor invasion in primary colorectal tumors ${ }^{\mathrm{a}}$

\begin{tabular}{|c|c|c|c|c|c|c|c|c|c|c|}
\hline & \multicolumn{2}{|c|}{ All patients } & \multicolumn{2}{|l|}{ Cases } & \multicolumn{2}{|l|}{ Controls } & \multirow[t]{2}{*}{$\mathrm{P}^{\mathrm{e}}$} & \multicolumn{3}{|c|}{ Univariate analysis } \\
\hline & Number & Percent & Number & Percent & Number & Percent & & OR & $95 \%$ CI & $P$ value \\
\hline \multicolumn{11}{|c|}{ BMVD invasive front $(n=90)^{\mathrm{b}, \mathrm{c}}$} \\
\hline Low & 76 & 84 & 24 & 75 & 52 & 90 & $0.077^{\mathrm{f}}$ & 1 & & \\
\hline High & 14 & 16 & 8 & 25 & 6 & 10 & & 2.9 & $(0.9-9.3)$ & 0.074 \\
\hline \multicolumn{11}{|c|}{ LMVD tumor stroma or invasive front $(n=91)^{\mathrm{b}, \mathrm{d}}$} \\
\hline Low & 72 & 79 & 22 & 67 & 50 & 86 & 0.027 & 1 & & \\
\hline High & 19 & 21 & 11 & 33 & 8 & 14 & & 3.1 & $(1.1-8.8)$ & 0.032 \\
\hline \multicolumn{11}{|c|}{ Blood vessel invasion $(n=96)$} \\
\hline Absent & 95 & 99 & 32 & 97 & 63 & 100 & $0.344^{\mathrm{f}}$ & NA & & \\
\hline Present & 1 & 1 & 1 & 3 & 0 & 0 & & & & \\
\hline \multicolumn{11}{|c|}{ Lymphatic vessel invasion $(n=96)$} \\
\hline Absent & 88 & 92 & 28 & 85 & 60 & 95 & $0.119^{\mathrm{f}}$ & 1 & & \\
\hline Present & 8 & 8 & 5 & 15 & 3 & 5 & & 3.6 & $(0.8-16.0)$ & 0.096 \\
\hline
\end{tabular}

$B M V D$ Blood microvessel density, LMVD lymphatic microvessel density, $O R$ odds ratio, $C I$ confidence interval, $N A$ not assessable

${ }^{a}$ Primary tumors from three controls could not be retrieved from the archive and tissue sections which were destroyed after staining were excluded from analysis leading to a variable number of examined slides; blood microvessels were stained with anti-CD31 antibodies and lymphatic vessels were stained with D2-40 antibodies

${ }^{\mathrm{b}}$ Maximum number of counted vessels from hot spots; invasive front was not present on all sections

${ }^{\mathrm{c}}$ High: greater than 46

${ }^{\mathrm{d}}$ High: greater than 20

${ }^{\mathrm{e}}$ Chi-square test of cases versus controls, unless mentioned otherwise

${ }^{\mathrm{f}}$ Fisher's exact test

cell morphology. Regions of necrosis were excluded from analysis.

Blood and lymphatic vessel invasion was assessed in HE stained sections and in sections stained with antibodies directed against CD31 or D2-40. In HE stained sections, the presence of tumor cells in luminal spaces lined by endothelial cells in peritumoral stroma was scored as blood or lymphatic vessel invasion. On immunohistochemically stained sections, the presence of tumor cells inside a CD31 or D2-40 stained vessel was considered as blood or lymphatic vessel invasion.

\section{Statistical Analysis}

Statistical analysis was carried out using SPSS software, version 12.0.1 (SPSS Inc, Chicago, IL). For both blood and lymphatic vessels, statistical analysis was performed using the maximum number of counted vessels in the hot spots as was also done in a recent study in which the same antibodies were used [20]. Numerical data are presented as mean \pm standard deviation or as median and range in case of skewness. The clinicopathologic features of cases and controls were compared either by a Chi-square test, Student $T$-test or Mann-Whitney test. Univariate and multivariate odds ratio's (OR), 95\% confidence intervals $(95 \% \mathrm{CI})$ and $p$-values $(\mathrm{P})$ were calculated by applying logistic regression analysis with patient's clinical outcome as dependent variable. Parameters with a (overall) p-value less than 0.10 in the univariate analysis were entered into the multivariate logistic regression model.

\section{Results}

\section{Standard Patient and Histopathological Characteristics}

No significant difference in sex, $\mathrm{T}$ stage, tumor size, tumor location, tumor differentiation, mucinous tumors, number of harvested lymph nodes and serum CEA level was seen between the case and control group. We did see that patients from the case group were significantly more often identified with tumors showing an infiltrative growth pattern in comparison to patients from the control group (Chi-square test, $41 \%$ versus $21 \%, P=0.031$ ). Patient characteristics are shown in Table 1 and univariate analysis of clinicopathological characteristics is shown in Table 4 .

\section{Expression of Sialyl Lewis X, VEGF-C and VEGF-D}

Membrane and cytoplasmic staining for CSLEX1 were scored separately. Membrane and cytoplasmic staining respectively was seen in 91 and 74 of 99 examined CSLEX1 stained sections. Significantly more cases than 
controls showed cytoplasmic staining for CSLEX1 (Chisquare test, $91 \%$ versus $67 \%, P=0.012$ ) (Table 2). No difference in membrane staining was seen between the case and control group.

More cases than controls were identified with cytoplasmic positively stained tumor cells for VEGF-C (Chi-square test, $44 \%$ versus $27 \%, P=0.093$ ) and VEGF-D (Fisher's exact test, $21 \%$ versus $6 \%, P=0.039$ ) (Table 2 ). Representative immunohistochemical stainings for CSLEX1, VEGF-C and VEGF-D are shown in Fig. 1. Cytoplasmic background staining was seen in negative controls for the VEGF-C staining. Tumors negative for VEGF-C showed similar background staining which could be clearly differentiated from positive staining for VEGF-C (Fig. 1e,f). The univariate analysis of these angiogenesis and lymphangiogenesisrelated tumor cell markers is shown in Table 2.

\section{Blood and Lymphatic Microvessel Density}

We choose to count blood and lymphatic microvessels in three hot spots (i.e. fields with the highest vascular density) located in intratumoral stroma and three hot spots at the

Table 4 Univariate analysis of clinicopathological characteristics

\begin{tabular}{|c|c|c|c|}
\hline \multirow[t]{2}{*}{ Characteristics } & \multicolumn{3}{|c|}{ Univariate analysis } \\
\hline & OR & $95 \% \mathrm{CI}$ & $P$ value \\
\hline \multicolumn{4}{|l|}{ Sex } \\
\hline Female & 1 & & \\
\hline Male & 1.3 & $(0.6-3.0)$ & 0.497 \\
\hline Age (years) & 1.0 & $(0.97-1.03)$ & 0.910 \\
\hline \multicolumn{4}{|l|}{ T stage } \\
\hline $\mathrm{T} 2$ and $\mathrm{T} 3$ & 1 & & \\
\hline $\mathrm{T} 4$ & 3.1 & $(1.0-9.9)$ & 0.050 \\
\hline Tumor size $(\mathrm{cm})(n=104)$ & 0.9 & $(0.8-1.2)$ & 0.536 \\
\hline \multicolumn{4}{|l|}{ Tumor location } \\
\hline Colon (coecum-sigmoid) & 1 & & \\
\hline Rectum (rectosigmoid-rectum) & 0.9 & $(0.3-2.6)$ & 0.858 \\
\hline Differentiation & & & $0.913^{\mathrm{a}}$ \\
\hline Good & 1 & & \\
\hline Moderate & 1.0 & $(0.4-2.5)$ & 0.927 \\
\hline Poor & 1.3 & $(0.3-4.9)$ & 0.750 \\
\hline Number of lymph nodes & 0.9 & $(0.9-1.0)$ & 0.132 \\
\hline \multicolumn{4}{|l|}{ Mucinous } \\
\hline No & 1 & & \\
\hline Yes & 1.4 & $(0.4-5.2)$ & 0.640 \\
\hline \multicolumn{4}{|l|}{ Growth pattern } \\
\hline Expanding or pushing & 1 & & \\
\hline Infiltrating & 2.7 & $(1.1-6.7)$ & 0.034 \\
\hline \multicolumn{4}{|c|}{ Preoperative serum CEA level $(n=43)$} \\
\hline$<6 \mathrm{ug} / 1$ & 1 & & \\
\hline$\geq 6 \mathrm{ug} / 1$ & 2.2 & $(0.5-10.2)$ & 0.303 \\
\hline
\end{tabular}

$O R$ Odds ratio, $C I$ confidence interval, $C E A$ carcinoembryonic antigen ${ }^{\text {a }}$ Overall $P$-value invasive front. Blood microvessels stained by anti-CD31 antibodies were seen in tumor stroma or the invasive front in all of 96 analyzed primary tumors $(100 \%)$ and lymphatic microvessels stained by D2-40 antibodies were seen in 78 of $96(81 \%)$ analyzed primary tumors. As previously shown $[21,22]$, we found that anti-CD31 antibodies did not stain D2-40 positive lymphatic vessels and vice versa (Fig. 2 ). We counted more blood microvessels $(39 \pm 12$; median 37 ; range 12 to 80$)$ than lymphatic microvessels ( $14 \pm 9$; median 15; range 0 to 42 ). Patients with more than 46 blood microvessels in the invasive front (high BMVD) were more often seen in the case than in the control group (Fisher's exact test, $25 \%$ versus $10 \%, P=0.077$ ), but it did not reach significance (Table 3 ). Significantly more patients with more than 20 lymphatic microvessels in tumor stroma or invasive front (high LMVD) were seen in the case group than in the control group (Chi-square test, $33 \%$ versus $14 \%$, $P=0.027$ ) (Table 3).

Concluding, overall more blood microvessels than lymphatic microvessels were observed. In the case group, a higher number of blood and lymphatic microvessels was seen compared to the control group.

\section{Blood and Lymphatic Vessel Invasion}

Blood and lymphatic vessel invasion detected on HE stained sections will be referred to as morphological vasoinvasion. In 49 of 108 (45\%) primary tumors, morphological vaso-invasion was found.

Lymphatic vessel invasion was seen in 8 of $96(8 \%)$ D240 stained tumor sections (Fig. 3a,b) and blood vessel invasion was only seen in one of $96(1 \%)$ sections stained with antibodies directed against CD31 (Fig. 3c,d). Seven of the 8 patients with lymphatic vessel invasion detected on the D2-40 stained sections had been recognized on the HE stained sections and the one tumor with blood vessel invasion was also seen on the HE stained section (Fig. 3g). These results show that morphological vaso-invasion scored on HE stained sections overestimated the actual blood and lymphatic vessel invasion as seen after immunohistochemical staining.

Relation between studied variables

We assessed mutual correlation between the different markers that were investigated. We found that 10 of 11 tumors showing positive staining for VEGF-D were also positive for VEGF-C (Chi-square test, $P<0.001$ ).

Significantly more patients with positively stained tumor cells for VEGF-C showed a high LMVD compared to patients negative for VEGF-C (Chi-square test, 11 of 31 versus 8 of $59, P=0.015$ ) indicating an association between VEGF-C expression and lymphangiogenesis. Sialyl Lewis 

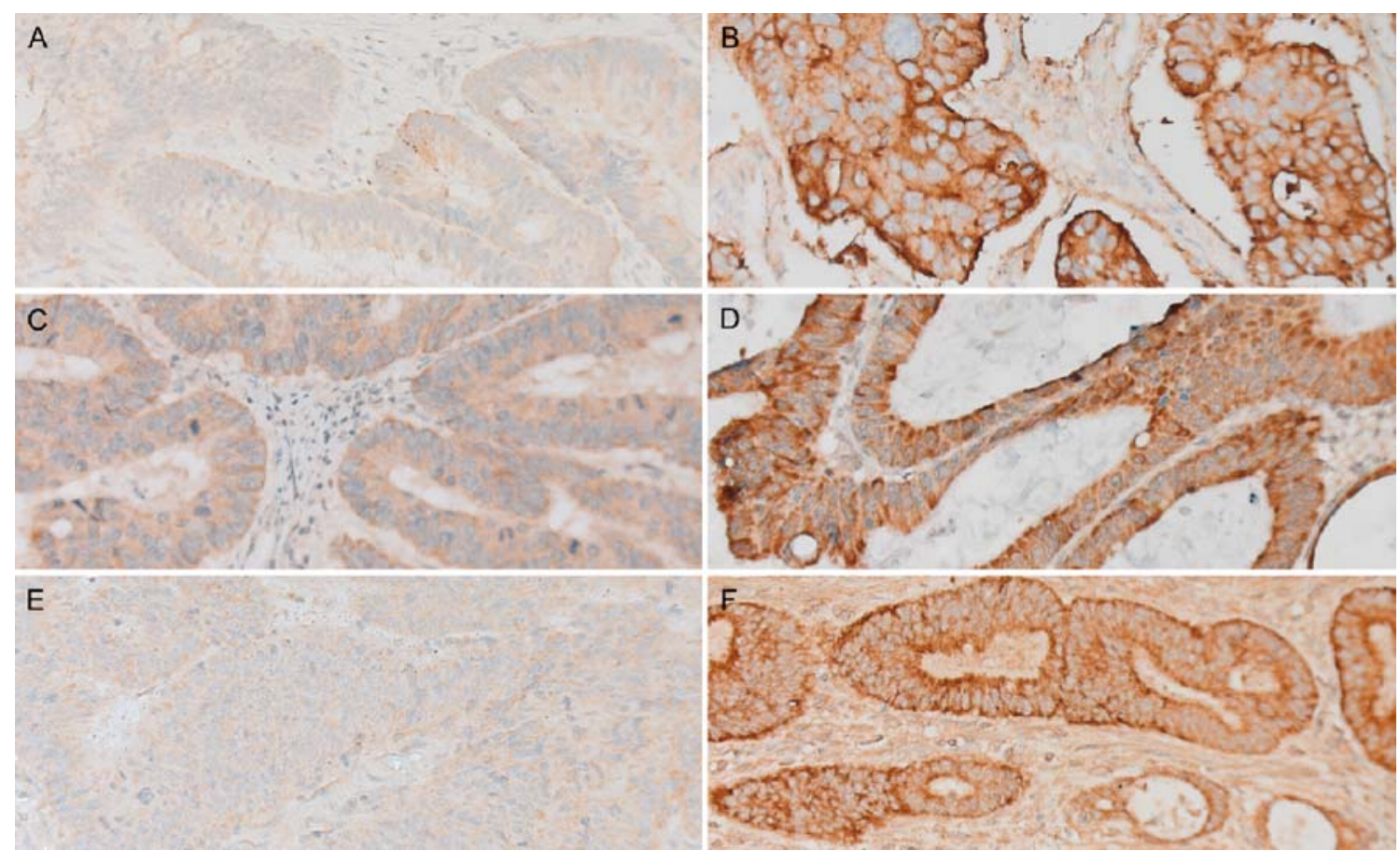

Fig. 1 CSLEX1, VEGF-C and VEGF-D expression in colorectal tumors. A tumor negatively stained (a) and positively stained (b) for CSLEX1; this tumor showed both membrane and cytoplasmic brown staining. A tumor with negative (c) and positive cytoplasmic brown staining (d) for VEGF-C; similar faint cytoplasmic brown staining as shown in negatively stained tumors for VEGF-C was seen in

phosphate buffered saline controles for the VEGF-C staining; this background staining could be clearly differentiated from positive cytoplasmic brown staining for VEGF-C. A tumor with negative (e) and positive cytoplasmic brown staining (f) for VEGF-D; a brown stromal background staining was seen

Fig. 2 Visualization of lymphatic and blood vessels in colorectal tumors. Lymphatic vessels were stained brown when using immunohistochemistry by the antibodies D2-40 (a). These lymphatic vessels showed no staining by immunohistochemistry when using antibodies CD31 (b). Blood vessels were not recognized by D2-40 (c) but were brown stained by CD31 (d)
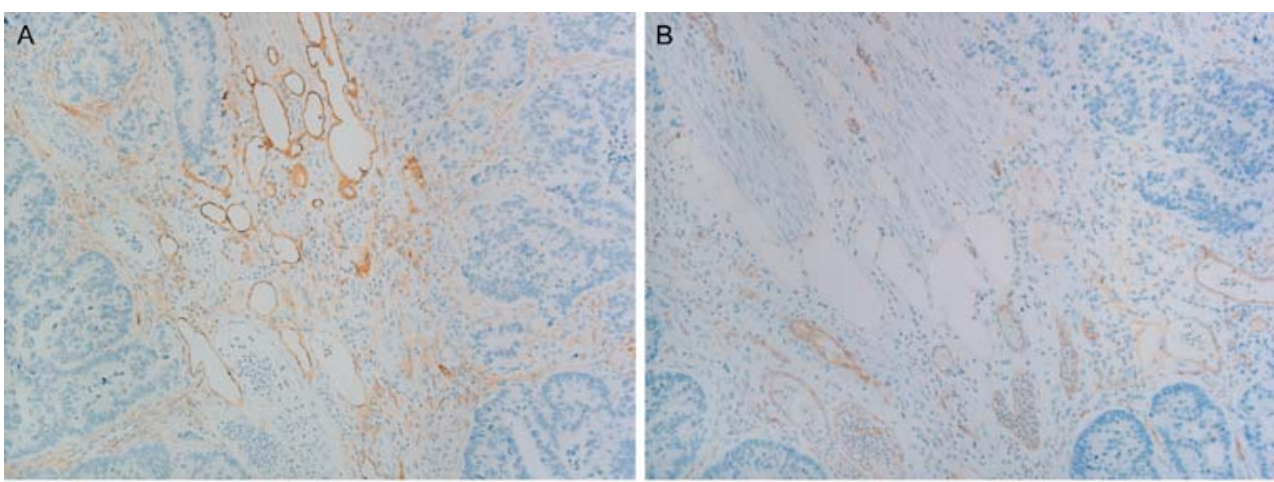

C

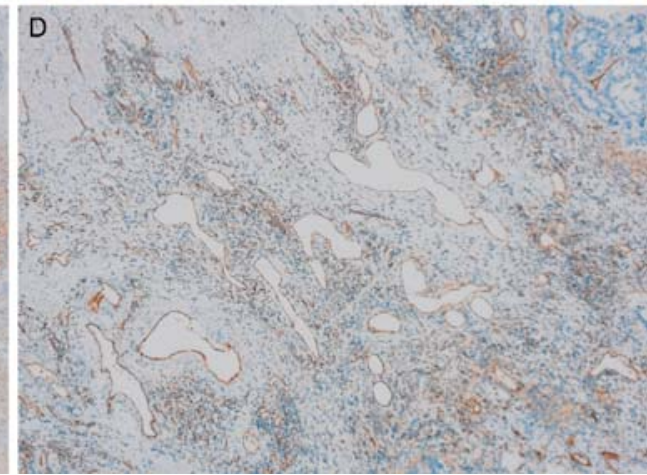


Fig. 3 Tumor cell invasion in lymphatic and blood vessels in colorectal tumors. Lymphatic vessel invasion seen on a hematoxylin and eosin stained section (a) and immunohistochemically stained section by the antibodies D2-40 (b); lymphatic vessels were brown stained by $\mathrm{D} 2-40$. Blood vessel invasion seen on a hematoxylin and eosin stained section (c) and immunohistochemically stained section by the antibodies CD31 (d); blood vessels were brown stained by CD31
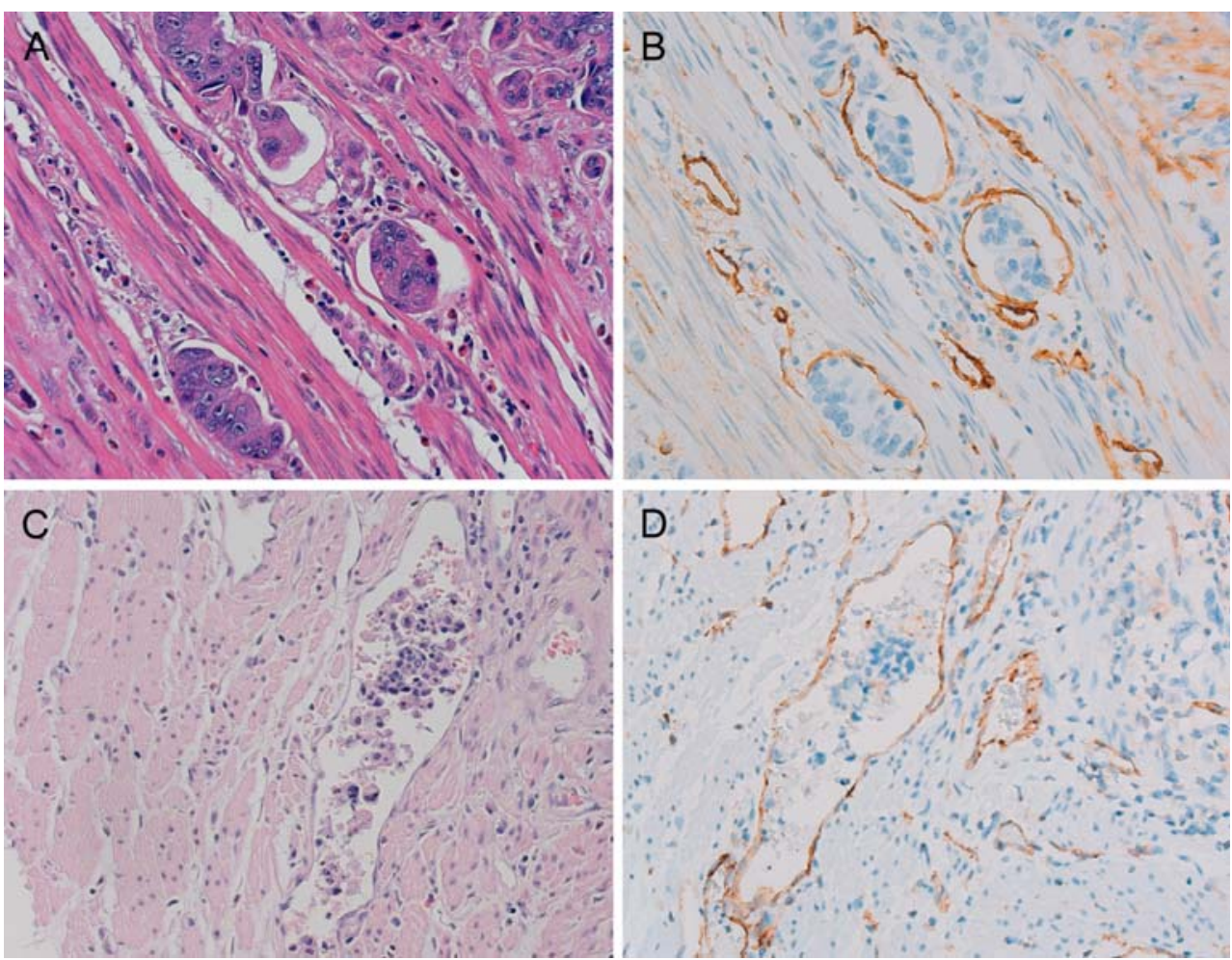

$\mathrm{X}$ expression seemed to be related to an infiltrating type of tumor growth as significantly more of these tumors showed cytoplasmic positive staining with CSLEX1 (Chi-square test, 24 of 27 versus 46 of $68, P=0.034$ ) compared to tumors with an expanding growth pattern. Also significantly more infiltrating tumors showed morphological vaso-invasion compared to expanding tumors (Chi-square test, 19 of 27 versus 27 of $70, P=0.005$ ). This correlation suggests a role of infiltrating tumor growth in the overestimation of blood and lymphatic vessel invasion in the morphological vasoinvasion score.

\section{Logistic Regression Analysis}

All variables with a $p$-value lower than 0.10 in the univariate analysis (Tables 2, 3 and 4) were entered in a forward step multivariate analysis. $\mathrm{T}$ stage, tumor growth pattern, CSLEX1-, VEGF-C- or VEGF-D-expression, high BMVD, high LMVD and lymphatic vessel invasion were entered in the multivariate analysis. The independent factors predicting disease recurrence that remained were sLeX tumor expression by CSLEX1 and a high LMVD (OR 5.1, 95\% CI 1.3-20.0 and OR 3.1, 95\% CI 1.0-10.0, respectively).

\section{Correlation with Site of Metastases}

The site of the metastases was registered in our hospital, which enabled us to assess its correlation with the different variables tested. Primary tumors with CSLEX1 expression significantly more often led to disease recurrence in the liver compared to primary tumors negative for CSLEX1 (Chi-square test, 20 of 74 versus one of $25, P=0.015$ ). Liver metastases were also significantly more often seen in the patients with infiltrative tumors than in patients with expanding tumors (Chi-square test, 11 of 27 versus 11 of $70, P=0.008)$.

Patients with a high BMVD in the invasive front developed lung metastases significantly more often than patients with a low BMVD in the invasive front (Fisher's exact test, 4 of 14 versus 4 of $76, P=0.018$ ).

The chance to develop regional, peritoneal or metastases to the brain, bones or adrenal glands was significantly higher in patients with high LMVD compared to patients with low LMVD (Fisher's exact test, 8 of 19 versus 8 of 72, $P=0.004)$. This chance also significantly increased with the T stage (Fisher's exact, T4 stage: 6 of 14 versus T2 or T3 stage: 12 of $94, P=0.012$ ) and decreased with the number of harvested lymph nodes (Fisher's exact test, 0 of 22 in patients with 12 or more examined lymph nodes versus 18 of 86 in patients with less than 12 lymph nodes, $P=$ $0.021)$. Moreover, no correlation was seen between a high LMVD, T4 stage or 12 or more harvested lymph nodes. When entering these three variables in a multivariate analysis, a high LMVD remained as an independent predictor for regional, peritoneal or metastases to distant sites other than liver and lungs (OR 7.3, 95\% CI 2.0-27.4, $P=0.003)$. 


\section{Discussion}

In this study, we show sLeX expression and a high LMVD of the primary tumor to be independent risk factors for disease recurrence in curatively resected CRC patients with tumor-negative lymph nodes. Our results confirm data from Nakagoe et al. [9] showing that lymph node-negative CRC patients with sLeX expression detected with CSLEX1 have a worse prognosis. Moreover, we showed a significant correlation between sLeX expression and liver metastases as previously reported by others $[23,24]$. We saw a correlation between sLeX expression and infiltrative tumor growth pattern and showed the latter also to correlate with disease recurrence [18]. The reproducibility of tumor growth pattern assessment has been shown to be problematic [25] which suggests growth pattern to be an unreliable prognostic marker in contrast with sLeX immunohistochemical detection. Our results suggest that sLeX expression plays a role in infiltrative tumor growth and in facilitating the hematogenous spread of tumor cells through blood microvessels via the portal vein to the liver.

The lymphatic system has also been believed to be one of the most important pathways for tumor cell dissemination as it is expected that tumor cells can enter lymphatic microvessels easier than blood microvessels because the former show a discontinuous or completely absent basement membrane and are devoid of pericytes [26]. Years of research have resulted in several lymphatic endothelial cell specific markers [26]. In this study, we used D2-40 which was reported to be more sensitive in detecting lymphatic endothelium than Prox1, LYVE-1 and podoplanin [27]. We found a high LMVD detected with D2-40 to be an independent risk factor for disease recurrence. Similar findings were seen by Matsumoto et al. [28] who used the antipodoplanin antibody to detect lymphatic microvessels in primary tumors of 106 stage I to IV CRC patients. They showed a high LMVD and lymphatic vessel invasion to correlate with a poor outcome but only the former remained as an independent predictor in the multivariate analysis. Saad et al. [20] examined BMVD and LMVD in 90 stage I to IV CRC patients by using anti-CD31 and D2-40 antibodies, respectively. They observed a significant correlation between LMVD and liver metastases, but they did not analyze other types of distant metastases. In our study, a high LMVD was found not to correlate with liver metastases or lung metastases but with regional intra-abdominal or intrapelvic metastases in lymph nodes and other distant metastases such as peritoneum, bones, brain and adrenal glands. We suggest that a high LMVD leads to tumor cell dissemination through lymphatic microvessels into intra-abdominal or pelvic lymph nodes. The lymphatic system finally returns lymph to the systemic blood circulation via the thoracic duct leading to metastases in the bones, brain and other distant sites.
Additionally, a high BMVD correlated with disease recurrence restricted to the lungs. This may explain the variability in published studies regarding the prognostic relevance of BMVD as it probably depends on the number of patients in those particular studies who suffered from disease recurrence in the lungs. Accordingly, a recent metaanalysis showed a high BMVD, detected by using antibodies directed against CD31 or CD34, to significantly predict poor clinical outcome [29].

We assessed tumor invasion in blood and lymphatic vessels on HE stained sections and referred to this as morphological vaso-invasion. By comparing morphological vaso-invasion and blood and lymphatic vessel invasion scored on immunohistochemically stained sections, we found the former to be overestimated as reported previously by others [30]. Analysis suggested that scoring of morphological vaso-invasion does not distinguish between blood or lymphatic vessel invasion and an infiltrative tumor growth pattern. Previous studies have shown that artifactual tissue retraction around tumor islands complicate and overestimate true lymphovascular invasion [16, 17]. Immunohistochemical staining of blood and lymphatic vessels with specific antibodies resolves this problem and enables objective estimation of tumor invasion in vessels.

To our knowledge, we are the first to identify a multiple set of tumor markers each correlating with a different preferential site of metastasis in CRC. Our results support two types of mechanisms involved in metastasis. First, there is the mechanical way of tumor cell dissemination through blood and lymphatic microvessels, resulting in tumor cell arrest in the narrow capillary network in different organs including lymph nodes. There they may proliferate and develop clinically evident metastases. Hematogenous and lymphatic metastasis is expected to increase with the number of blood and lymphatic microvessels. This may explain the correlation between a high BMVD and lung metastases and the correlation between a high LMVD and regional intra-abdominal or intrapelvic metastases in lymph nodes with eventually distant metastases.

In addition to this mechanical entrapment of tumor cells, also biological processes determine outgrow of metastases as explained by the "seed and soil" theory [31, 32]. This theory suggests that a subpopulation of tumor cells with metastatic potential, recently identified as colon-cancerinitiating cells [33], disseminate through the whole body. These cells proliferate and differentiate to form clinically evident metastases at preferential sites depending on local molecular interaction among which availability of local growth factors. In our study, the correlation between sLeX expression and liver metastases may be explained by this pathway. The interaction of the antigen sLeX on tumor cells and E-selectin on endothelial cells was shown to mediate 
adhesion of tumor cells to endothelial cells [8] possibly facilitating tumor cell invasion in blood microvessels, extravasation and migration into tissue. Additionally, colorectal tumor cells showing sLeX expression might prefer the liver to grow out to form clinically evident metastases due to interaction with local E-selectin [34].

It is important to realize that this study regards a small patient group but even in these small patient numbers we showed significant findings consistent with current thoughts in terms of hematogenous and lymphatic metastasis. Furthermore, only $20 \%$ of our patient group had undergone an adequate lymph node harvest of at least 12 lymph nodes as recommended by the American Joint Committee on Cancer Staging [35], which is a limitation of our study. Nevertheless, similar numbers were reported by Johnson et al. [36] and Baxter et al. [37] who showed adequate lymph node evaluation in $22 \%$ of 569 CRC specimens respectively $37 \%$ of 116995 CRC patients. They reported the effect of different pathology assistants, older age and rectal cancer to affect the number of lymph nodes retrieved [37, 38].

In summary, our study shows that high-risk lymph nodenegative CRC patients can be identified by assessing the primary tumor for sLeX expression with CSLEX1 and LMVD. Our results have to be validated in prospective studies which should also evaluate whether these high-risk CRC patients may benefit from adjuvant systemic therapy. Moreover, our results are consistent with the notion that both a lymphatic and hematogenous route plays a role in CRC.

Acknowledgments The authors would like to thank Jan Molenaar, Department of Oncology Documentation, for providing patient's data from the cancer registry ONCDOC and Connie Janssen-van Rhijn and Rob Keyzer, Department of Surgery, for their technical assistance.

\section{References}

1. Moertel CG, Fleming TR, Macdonald JS et al (1990) Levamisole and fluorouracil for adjuvant therapy of resected colon carcinoma. N Engl J Med 322:352-358

2. Impact B2 Investigators (1999) Efficacy of adjuvant fluorouracil and folinic acid in B2 colon cancer. International Multicentre Pooled Analysis of B2 Colon Cancer Trials (IMPACT B2) Investigators. J Clin Oncol 17:1356-1363

3. Madbouly KM, Senagore AJ, Mukerjee A et al (2007) Does immunostaining effectively upstage colorectal cancer by identifying micrometastatic nodal disease? Int J Colorectal Dis 22:39-48

4. Cianchi F, Palomba A, Boddi V et al (2002) Lymph node recovery from colorectal tumor specimens: recommendation for a minimum number of lymph nodes to be examined. World J Surg 26:384-389

5. Quasar Collaborative Group, Gray R, Barnwell J et al (2007) Adjuvant chemotherapy versus observation in patients with colorectal cancer: a randomised study. Lancet 370:2020-2029

6. Liotta LA, Stetler-Stevenson WG (1991) Tumor invasion and metastasis: an imbalance of positive and negative regulation. Cancer Res 51:5054s-5059s
7. Phillips ML, Nudelman E, Gaeta FC et al (1990) ELAM-1 mediates cell adhesion by recognition of a carbohydrate ligand, sialyl-Lex. Science 250:1130-1132

8. Walz G, Aruffo A, Kolanus W et al (1990) Recognition by ELAM-1 of the sialyl-Lex determinant on myeloid and tumor cells. Science 250:1132-1135

9. Nakagoe T, Fukushima K, Tanaka K et al (2002) Evaluation of sialyl Lewis(a), sialyl Lewis(x), and sialyl Tn antigens expression levels as predictors of recurrence after curative surgery in nodenegative colorectal cancer patients. J Exp Clin Cancer Res $21: 107-113$

10. Grabowski P, Mann B, Mansmann U et al (2000) Expression of SIALYL-Le(x) antigen defined by MAb AM-3 is an independent prognostic marker in colorectal carcinoma patients. Int $\mathrm{J}$ Cancer 88:281-286

11. Li X, Eriksson U (2001) Novel VEGF family members: VEGF-B, VEGF-C and VEGF-D. Int J Biochem Cell Biol 33:421-426

12. Veikkola T, Karkkainen M, Claesson-Welsh L et al (2000) Regulation of angiogenesis via vascular endothelial growth factor receptors. Cancer Res 60:203-212

13. Stacker SA, Caesar C, Baldwin ME et al (2001) VEGF-D promotes the metastatic spread of tumor cells via the lymphatics. Nat Med 7:186-191

14. Akagi K, Ikeda Y, Miyazaki M et al (2000) Vascular endothelial growth factor-C (VEGF-C) expression in human colorectal cancer tissues. Br J Cancer 83:887-891

15. Weidner N, Semple JP, Welch WR et al (1991) Tumor angiogenesis and metastasis-correlation in invasive breast carcinoma. N Engl J Med 324:1-8

16. Hoda SA, Hoda RS, Merlin S et al (2006) Issues relating to lymphovascular invasion in breast carcinoma. Adv Anat Pathol 13:308-315

17. Algaba F (2006) Lymphovascular invasion as a prognostic tool for advanced bladder cancer. Curr Opin Urol 16:367-371

18. Jass JR, Love SB, Northover JM (1987) A new prognostic classification of rectal cancer. Lancet 1:1303-1306

19. Gundersen HJ, Bendtsen TF, Korbo L et al (1988) Some new, simple and efficient stereological methods and their use in pathological research and diagnosis. APMIS 96:379-394

20. Saad RS, Kordunsky L, Liu YL et al (2006) Lymphatic microvessel density as prognostic marker in colorectal cancer. Mod Pathol 19:1317-1323

21. Yonemura Y, Endou Y, Tabachi K et al (2006) Evaluation of lymphatic invasion in primary gastric cancer by a new monoclonal antibody, D2-40. Hum Pathol 37:1193-1199

22. Fukunaga M (2005) Expression of D2-40 in lymphatic endothelium of normal tissues and in vascular tumours. Histopathology 46:396402

23. Ichikawa D, Kitamura K, Tani N et al (2000) Molecular detection of disseminated cancer cells in the peripheral blood and expression of sialylated antigens in colon cancers. J Surg Oncol 75:98-102

24. Nakamori S, Kameyama M, Imaoka S et al (1993) Increased expression of sialyl Lewisx antigen correlates with poor survival in patients with colorectal carcinoma: clinicopathological and immunohistochemical study. Cancer Res 53:3632-3637

25. Deans GT, Heatley M, Anderson N et al (1994) Jass' classification revisited. J Am Coll Surg 179:11-17

26. Reis-Filho JS, Schmitt FC (2003) Lymphangiogenesis in tumors: what do we know? Microsc Res Tech 60:171-180

27. Van der Auwera I, Van den Eynden GG, Colpaert CG et al (2005) Tumor lymphangiogenesis in inflammatory breast carcinoma: a histomorphometric study. Clin Cancer Res 11:7637-7642

28. Matsumoto K, Nakayama Y, Inoue Y et al (2007) Lymphatic Microvessel Density is an Independent Prognostic Factor in Colorectal Cancer. Dis Colon Rectum 50:308-314 
29. Des Guetz G, Uzzan B, Nicolas P et al (2006) Microvessel density and VEGF expression are prognostic factors in colorectal cancer. Meta-analysis of the literature. Br J Cancer 94:18231832

30. Urabe A, Matsumoto T, Kimura M et al (2006) Grading system of lymphatic invasion according to D2-40 immunostaining is useful for the prediction of nodal metastasis in squamous cell carcinoma of the uterine cervix. Histopathology 49:493-497

31. Paget S (1989) The distribution of secondary growths in cancer of the breast. 1889. Cancer Metastasis Rev 8:98-101

32. Fidler IJ (1999) Critical determinants of cancer metastasis: rationale for therapy. Cancer Chemother Pharmacol 43(Suppl): S3-10

33. Ricci-Vitiani L, Lombardi DG, Pilozzi E et al (2007) Identification and expansion of human colon-cancer-initiating cells. Nature 445:111-115
34. Gout S, Tremblay PL, Huot J (2008) Selectins and selectin ligands in extravasation of cancer cells and organ selectivity of metastasis. Clin Exp Metastasis 25:335-344

35. Nelson H, Petrelli N, Carlin A et al (2001) Guidelines 2000 for colon and rectal cancer surgery. J Natl Cancer Inst 93:583-596

36. Johnson PM, Malatjalian D, Porter GA (2002) Adequacy of nodal harvest in colorectal cancer: a consecutive cohort study. J Gastrointest Surg 6:883-888

37. Baxter NN, Virnig DJ, Rothenberger DA et al (2005) Lymph node evaluation in colorectal cancer patients: a population-based study. J Natl Cancer Inst 97:219-225

38. Ostadi MA, Harnish JL, Stegienko S et al (2007) Factors affecting the number of lymph nodes retrieved in colorectal cancer specimens. Surg Endosc

39. Sobin LH, Wittekind C (2002) UICC TNM Classification of Malignant Tumours. Wiley, New York 